JOURNAL DE PHYSIQUE

Colloque C2, supplément au $\mathrm{n}^{\circ} 3$, Tome 47, mars 1986

page $c 2-473$

\title{
A NEW DETECTION SYSTEM FOR AN ATOM PROBE FIM
}

\author{
D. BLAVETTE, A.BOSTEL and J.M. SARRAU \\ Laboratoire de Microscopie Ionique, UA CNRS 808, UER SCiences \\ de Rouen, BP 67, F-76130 Mont-Saint-Aignan, France
}

Résumé - Un nouveau système de détection permettant de discriminer les ions de même temps de vol dans une sonde atomique est décrit. Quelques résultats préliminaires sont donnês et montrent les performances de la multidétection spatiale.

Abstract - A new detection technique for an atom-probe FIM is described. With such a system, ions having the same time of flight can be discriminated. Some preliminary results are given and show the performances of the spatial multidetection.

\section{I - INTRODUCTION}

It is well known that the field evaporation rate has a strong influence on the quantitative micro-analysis of materials by atom-probe techniques. When two or several ions of the same mass to charge ratio are field evaporated on a single pulse, they may strike simultaneously the detector. As no amplitude measurement of the detection signal is avalaible for deriving the number of ions striking the channel plate, measured compositions become erroneous.

These rate dependent effects are particulary pronounced when energy deficits are compensated or in case a high resolution pulse laser system is used /1/. Tsong /2/ was the first to propose a method of correction by assuming that the number of ions per pulse follows a Poisson distribution. However, the approximation used is no longer valid when analyses are taken on a low-index pole. Moreover, the dead time of the detection system as well as the distribution of ion energy deficits were not taken into account in the author's approach.

of course, corrections based on the isotopic distribution of atoms can be applied to concentrations to partially compensate for these detection artifacts. However, this confines the performances of real atom-probes. Indeed, preferential evaporation effects are frequently rate dependent specially when the evaporation of surface atoms is promoted by adsorption of reactive gas /3/. Higher analysis rates are therefore desirable. This paper is aimed at describing a new detection system which allows to get higher analysis rates with reduced effects of "pile-up".

\section{1 - EXPERIMENTAL}

The principle of the new system we developped is based on a spatial detection of ions. The detector is divided in four independant sectors with equal areas (fig. 1). With such a system, analyses can be performed four times faster with the same systematic error rate. For the sake of simplicity and compatibility the basic 
digital timer structure was preserved. A special electronic device has been designed for coding the ion position on the four sectors and supplying a classical detection signal. The latter is built from the logical sum $(\Sigma)$ of signals supplied by the four elementary detectors. Up to four times of flight can be recorded for each evaporation pulse in this preliminary version of the system. For each time event, the position of ions striking simuttaneously the detectors is stored in four 4-bit shift registers.

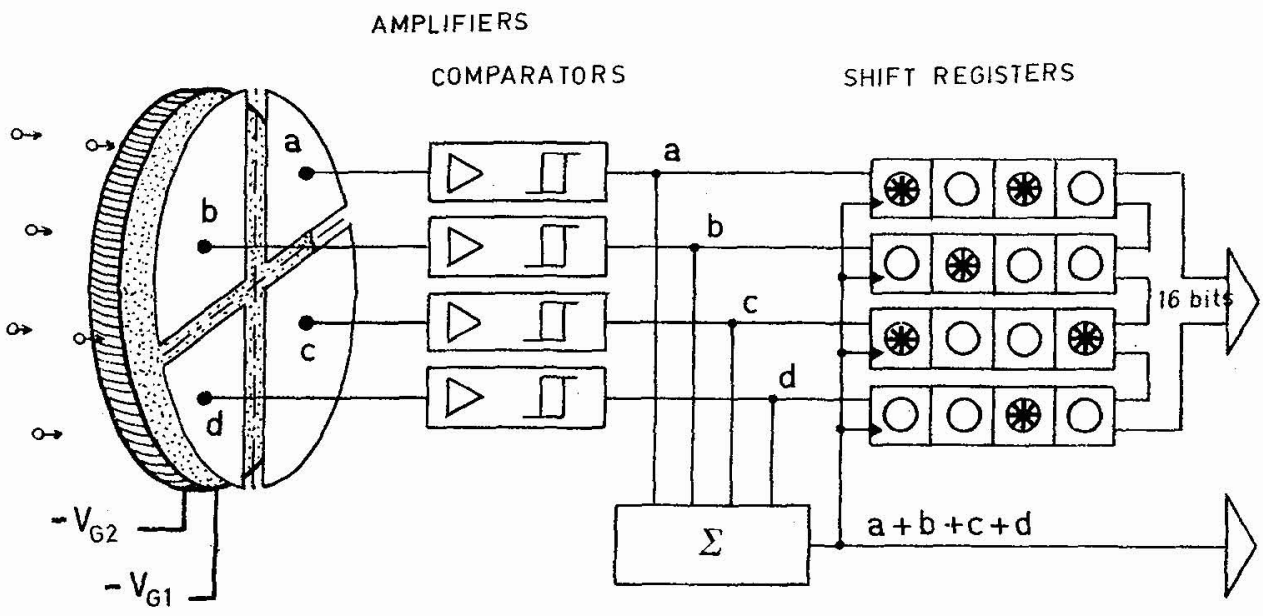

DIGITAL TIMERS

Fig. 1 - Principle of the four-sector detection system

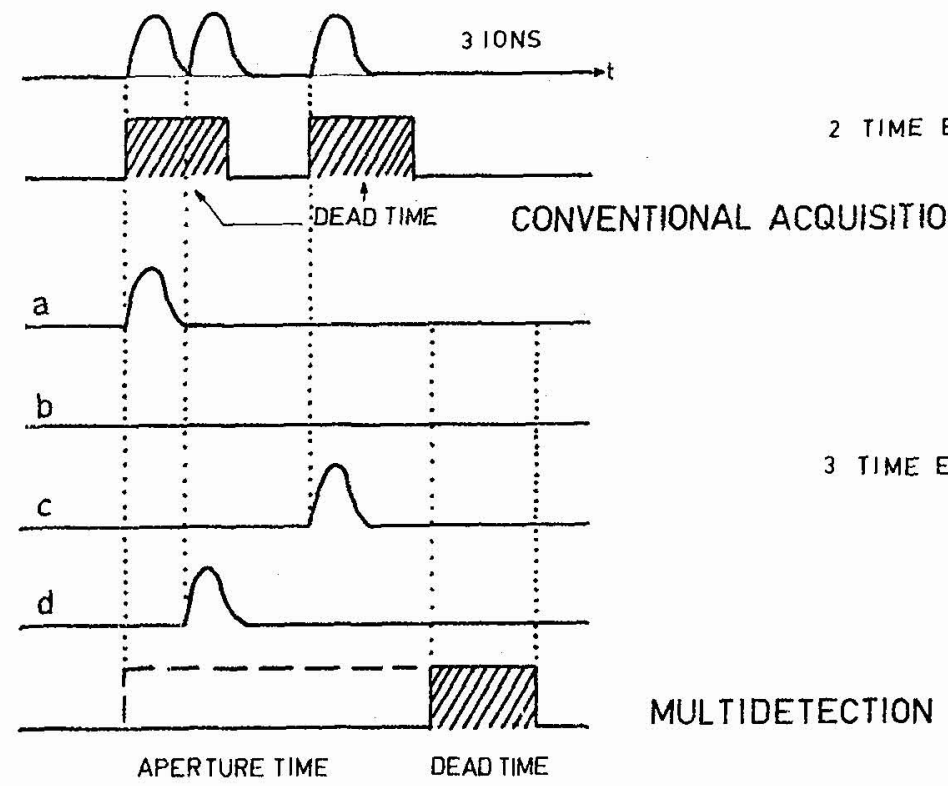

Fig. 2 - Detection signals and dead time in the multidetection system 
When no ion is received in a given sector a low logic level (0) is stored in the corresponding register and vice-versa. For each novel time event the register is shifted. This asynchronous system is reset when ion arrival is no longer expected.

This four-sector system has an important particularity (fig. 2). While standart detection systems are sensitive again after a certain dead time (5 ns) after the first ion, on the contrary, the multidetection remains operative during few nanoseconds ( 7 ns) after the first time event then is insensitive during a given time $(<5$ ns $)$. Ions received during the opening time are considered as having the same time of flight. This means that the shape of mass spectra is a little bit different of that usually observed with standard detection systems. In case a high resolution atom-probe is used, this opening time should be reduced to a value corresponding to the mass resolution. By choosing properly the opening time, this detection method is more efficient because most of ions are detected with apparent mass very close to the peak maximum in the spectra. In standard conditions, a simple calculation based on the observed exponential decay of spectra obtained with conventionnal atom-probes shows that nearly $80 \%$ of ions are detected within 10 ns $(M=30$, $V=10 \mathrm{kV}, L=1 \mathrm{~m}$ ). With high resolution atom-probes, this effect is much more pronounced so that the effective efficiency should be much better in this case.

In spite of its apparent simpicity, good operating conditions of this system are some what fastidious to adjust. For instance, the number of ions collected (i.e. the detection efficiency) must be equal for each sector. A carrefui adjustement of the four HF detection amplifier threshold is therefore necessary. On the other hand, propagation times must be equal for each channel. The connection lengths between each sector and the multidetection system have therefore to be carrefully settled.

In order to simplify the connection structure at the detector proximity, the four electron collectors of the channel plate have been grounded while, the front panel has been biased to a negative high voltage. With such a configuration, the shape of detector pulses is very weakly alterated. In addition thin tantalum foils have been used in order to prevent cross-talk from interfering between adjacent sectors. New wide band preamplifiers (>600 MHz) with very little back ground noise and hight dynamic sensitivity $(>40 \mathrm{~dB}$ ) have been developped. Because of their very little size, it has been possible to place the amplifier/comparators close by the four sectors in order to increase the noise immunity as well? as to preserve the pulse shape. The half maximum width of the calibrated signals supplied by the comparators (ECL) is 1.5 ns.

Acceleration of ions near the front panel of the channel plate was observed. This effect can however be compensated by calculating the apparent time of flight of ions as a function of the front panel polarisation.

\section{RESULTS}

Preliminary experiments have been carried out on a $\mathrm{Ni}_{3} \mathrm{Fe} \mathrm{Al}$ alloy in order to adjust the amplifier sensibility, control the cross-talk ratio, check the data reliability. Figure 3 shows four mass spectra built from ions detected on each sector. Before very thin electron collectors have been used a lot of multiple ions were erroneousty detected because of capacitor effects between adjacent sectors. As mass spectra show, the number of collected ions is nearly the same for each elementary detector. Compositions and detection rates are listed in Table 1.

\begin{tabular}{|c|c|c|c|c|c|}
\hline Sector number & $\begin{array}{l}\text { Number of coi- } \\
\text { lected ions }\end{array}$ & Detection rates $\%$ & at \% Al & at \% Fe & at \% Ni \\
\hline 1 & 1097 & 28.0 & 4.6 & 20.9 & 74.5 \\
2 & 925 & 23.6 & 3.0 & 23.3 & 73.7 \\
3 & 943 & 24.1 & 4.1 & 18.0 & 77.9 \\
4 & 954 & 24.3 & 3.9 & 21.7 & 74.4 \\
\hline
\end{tabular}

Table 1 - Detection rates for each detection sector 
In spite of careful adjustments, a little difference remains between the first sector efficiency and the other section ones. We think that this weak difference is caused by some assymetry in the four sector geometry. Compositions 1 isted in table 1 show that atom-probe data are similar for each sector and are close to the alloy stoichiometry.
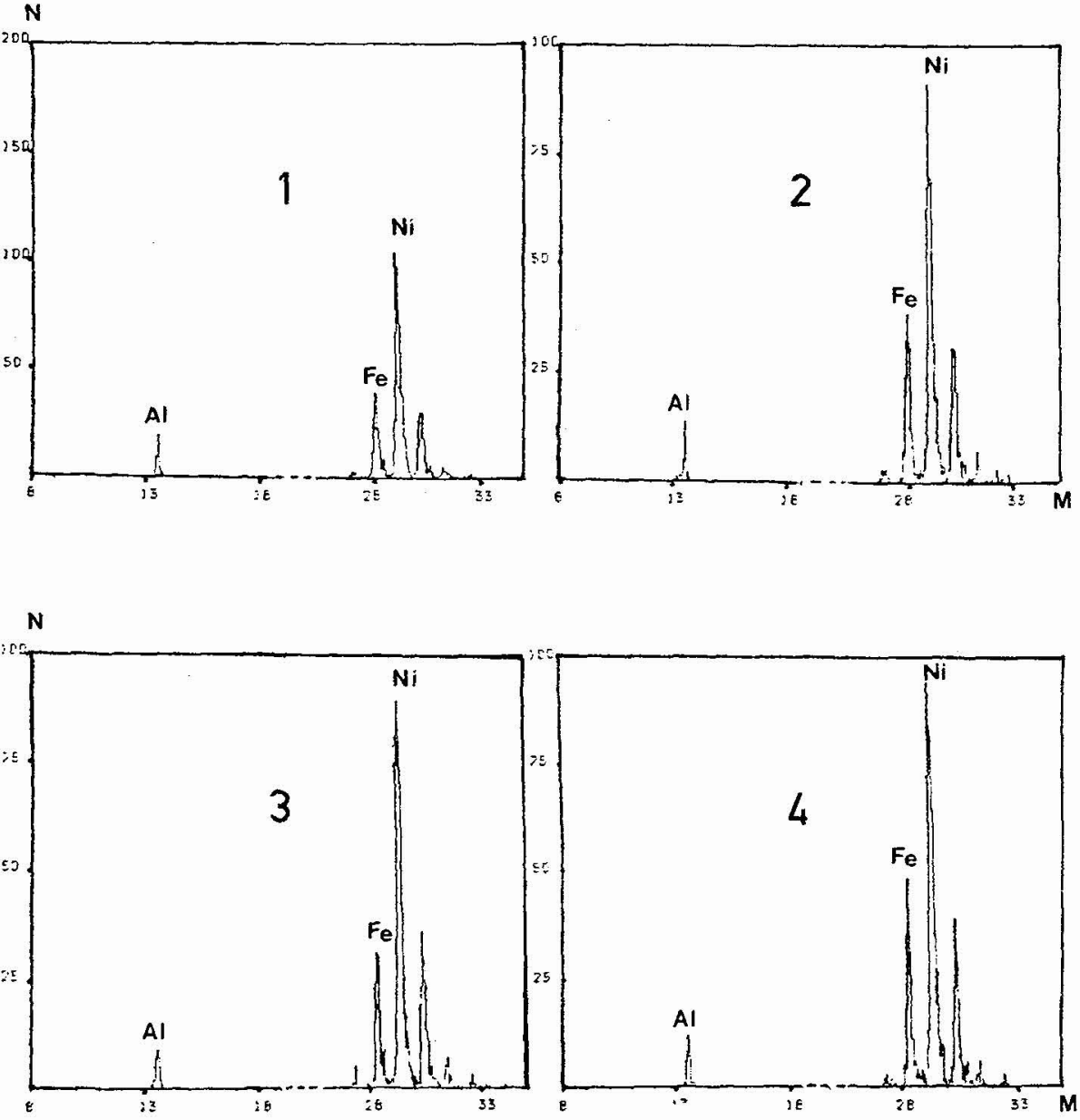

Fig. 3 - Atom-probe mass spectra obtained from each detection sector

In order to test the system ability to detect multiple events, we have performed analyses near the central portion of a low-index plane of the $\mathrm{Ni}_{3} \mathrm{Fe} A \mathrm{l}$ alloy. Table 2 summarizes the results we obtained. Numbers of collected ions of each species ( $\mathrm{Al}, \mathrm{Fe}, \mathrm{Ni}$ ) are given respectively in the standard detection and multidetection modes. In the former case, ions detected with the same time of flight are considered as one atom while in the latter mode the number of excited sectors is taken into account. As expected, a Targe amount of lost ions having the same apparent mass are retrieved when position data of ions are used. The results demonstrate that more than $10 \%$ of received ions are lost. 
Compositions are of course strongiy affected in the standard detection mode because of the high evaporation rate. The $\mathrm{Fe} / \mathrm{Ni}$ ratio is $33 \%$ in this latter mode while it is $30 \%$ when using the multidection. Concentrations are however a bit different from the alloy composition because of occurence of few preferential evaporation effects. As position data of the multidetection system were not yet "on line" connected to the computer, experiments were carried out with a low pulse repetition rate. As a result, a small proportion of nickel atoms was preferentialily evaporated at the D.C voltage between evaporation pulses.

\begin{tabular}{|l|c|c|c|c|}
\hline & AT & $\mathrm{Fe}$ & $\mathrm{Ni}$ & $\mathrm{Fe} / \mathrm{Ni}$ \\
\hline $\begin{array}{l}\text { Ions collected with the } \\
\text { multidetection } \\
N_{t}=1132\end{array}$ & $\begin{array}{c}65 \\
(5.7 \%)\end{array}$ & $\begin{array}{c}249 \\
(22.0 \%)\end{array}$ & $\begin{array}{c}818 \\
(72.3 \%)\end{array}$ & $30.4 \%$ \\
\hline $\begin{array}{l}\text { Ions collected with the } \\
\text { standard detection } \\
N_{\mathrm{t}}=1023\end{array}$ & $\begin{array}{c}64 \\
(6.2 \%)\end{array}$ & $\begin{array}{c}236 \\
(23.1 \%)\end{array}$ & $\begin{array}{c}723 \\
(70.7 \%)\end{array}$ & $32.7 \%$ \\
\hline
\end{tabular}

Table 2 - Comparison between the two detection modes

\section{CONCLUSION}

The preliminary results we obtained clearly demonstrate that a not negligible proportion of ions, which should not have been detected otherwise with standard detection systems, are retrieved with the new multidetector. The system described in this paper is a very preliminary version of a more versatile prototype we plane to develop. We think that this multidetection system will lead to decisive improvements in quantitative compositional analyses of materials by atom probe techniques specialiy in case plane by plane investigations are achieved.

An indirect consequence of the spatial detection is that informations can be drawn from composition profiles supplied by each detection sector. This facility can be of great interest for instance when phase or grain boundaries are crossed by the analysis cylinder.

\section{REFERENCES}

/1/ Cerezo, A., Smith, G. D. W. and Waugh, A. R., Proc. of the 31 st IFES, Journal de Physique C9-45 (1984) 329

12/ Tsong, T. T., Yee, S. Ng and Krishnaswamy S. V., App1. Phys. Lett. 32 (1978) 778

/3/ Wada, M., Vemori, R., Nishikawa, 0., Surf. Sci. 134 (1983) 17

\section{Acknowledgement}

The authors wish to thank the ANVAR (Agence Nationale de Valorisation de la Recherche) for its financial support. 